\title{
Progressive multifocal exophytic pontine glioblastoma: a case report with literature review
}

\author{
Fanfan Chen ${ }^{1}$, Zongyang Li ${ }^{2}$, Chengyin Weng ${ }^{3}$, Peng Li ${ }^{1}$, Lanbo Tu${ }^{1}$, Lei Chen ${ }^{2}$, Wei Xie ${ }^{1}$ and Ling Li4 ${ }^{*}$
}

\begin{abstract}
Multifocal pontine glioblastoma exhibiting an exophytic growth pattern in the cerebello-pontine angle (CPA) is rare. We present a case of a 5-year-old girl with consecutive neurological imaging and other clinical findings indicating progressive multifocal exophytic pontine glioblastoma. Three lesions were reported, of which two were initially presented, and one was developed 2 months later. One lesion demonstrated a progressing exophytic extension in the cistern of the left side of the CPA. The other two lesions were located and confined within the pons. Initial magnetic resonance imaging and positron emission tomography-computed tomography indicated low-grade glioma or inflammatory disease. However, 2 and 3 months later, subsequent magnetic resonance spectroscopy (MRS) displayed elevated choline and depressed $\mathrm{N}$-acetyl aspartate peaks compared with the peaks on the initial MRS, indicating a high-grade glioma. Subtotal resection was performed for the CPA lesion. Histopathologic examination showed discrepant features of different parts of the CPA lesion. The patient received no further chemotherapy or radiotherapy and died 2 months after surgery. The multifocal and exophytic features of this case and the heterogeneous manifestations on neurological images were rare and confusing for both diagnosis and surgical decision-making. Our case report may contribute knowledge and helpful guidance for other medical doctors.
\end{abstract}

Keywords: Brainstem, Cerebello-pontine angle, Glioma, Multiple lesion, Pontine

\section{Background}

Although brainstem tumors account for $10 \%-20 \%$ of cerebral neoplasms in children and $90 \%$ of them are gliomas $[1,2]$, exophytic pontine glioma located in the cerebellopontine angle (CPA) is uncommon [3-6]. Because of their heterogeneous biological behaviors and neurological imaging manifestations, the classification of brainstem gliomas is not consistent. Diagnosis and therapeutic decision-making are often difficult for gliomas, especially in the case of rare neurological imaging features.

Here, we report a special case of multifocal glioblastoma derived from the pons, exhibiting an exophytic growth pattern, in a 5-year-old girl. In total, three lesions were revealed in the posterior fossa. Repeated magnetic

\footnotetext{
*Correspondence: ling7810@126.com

${ }^{4}$ Record Department, Guangzhou First People's Hospital, Guangzhou

Medical University, Guangzhou 510180, Guangdong, P. R. China

Full list of author information is available at the end of the article
}

resonance imaging (MRI) showed the progression of the lesions in the left side of the CPA and the upper left side of the pons as well as the appearance of a new lesion in the right side of the pons. Furthermore, repeated magnetic resonance spectroscopy (MRS) over 3 months revealed a gradually elevated ratio of choline (Cho) to $\mathrm{N}$-acetyl aspartate (NAA).

\section{Case report \\ Medical history and examination}

The 5-year-old girl was admitted to the Neurosurgery Department of Guangzhou First People's Hospital, Guangzhou, China on October 6, 2015 and presented with a 2 month history of a medial gaze of the left eye. She did not complain of headache, dizziness, dysphagia, or vomiting. The outpatient medical record revealed that the patient frequently had fevers and coughs since the age of three. The last fever and cough were reported 1 week before the admission. The patient 
showed age-appropriate physical development. Cardiopulmonary auscultation results were normal. A neurological examination revealed left cranial VI nerve palsy without involvement of other nerves such as the ocular motor (cranial III or IV), trigeminal, facial, and acoustic nerves. No cerebellar signs or hemiparesis were present. The patient showed no signs of ataxia or long tract dysfunction.

Gadolinium-enhanced magnetic resonance imaging (MRI) was performed 2 days after the admission, demonstrating two well-defined, ring-enhanced lesions at the left side of the CPA $(2.0 \mathrm{~cm} \times 2.6 \mathrm{~cm}$; Fig. $1 \mathrm{~A})$ and upper left side of the pons $(0.6 \mathrm{~cm} \times 0.5 \mathrm{~cm}$; Fig. 1B). The MRI manifestation of the CPA lesion was similar to an extra-axial mass, e.g., nerve sheath tumor. The pons was obviously enlarged and swollen. MRS displayed a simultaneous decrease in $\mathrm{N}$-acetyl aspartate (NAA) and choline (Cho) (Fig. 1C). The Cho:NAA ratio was 0.82, and the Cho:Cr (creatine) ratio was 13.01. A peak at 1.3 part per million (ppm) was observed. Positron emission tomography-computed tomography (PET-CT) was performed, which demonstrated hypometabolic foci in the left side of the CPA, indicating low-grade glioma or inflammation (Fig. 1D).
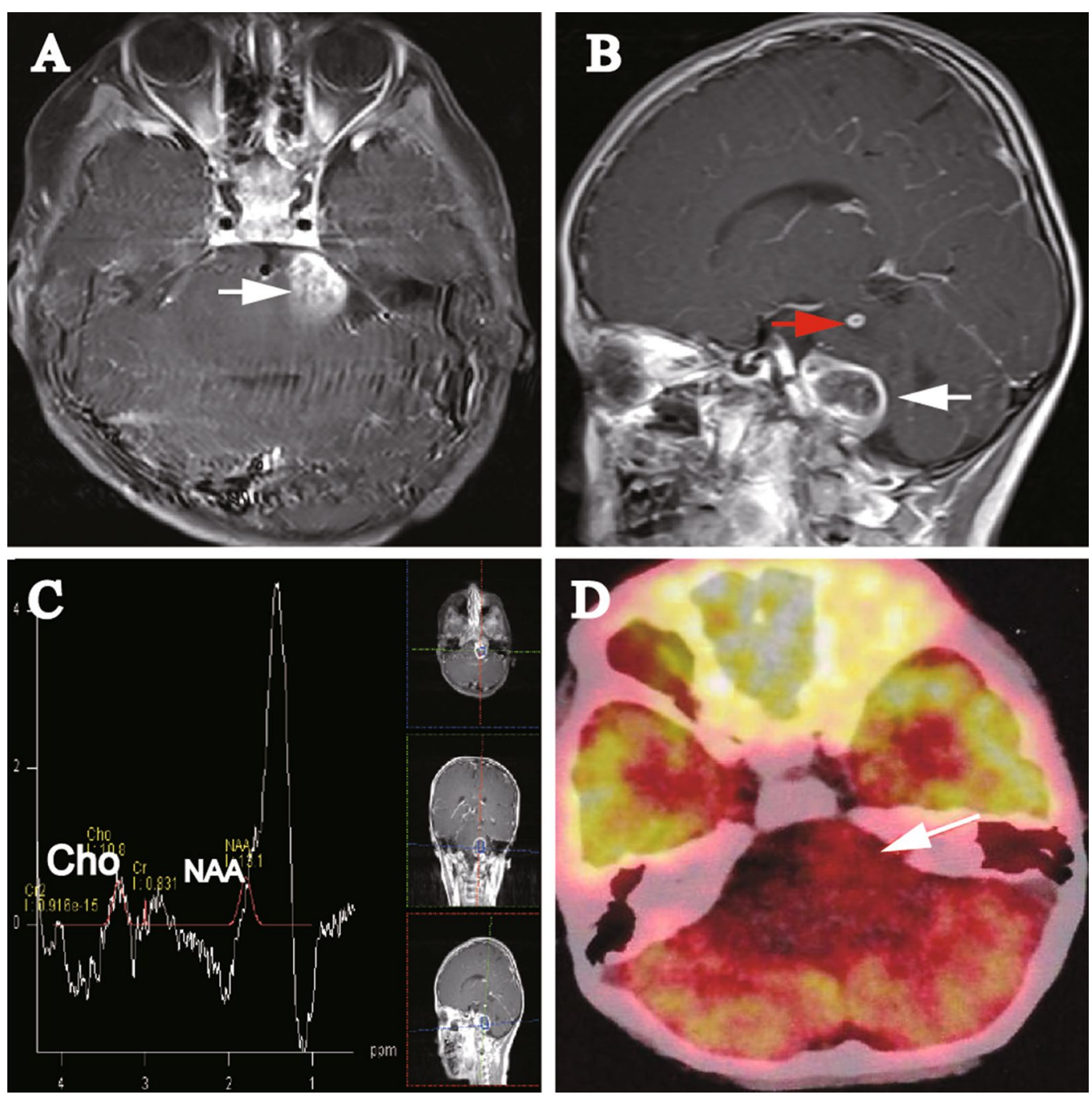

Fig. 1 Images of the initial magnetic resonance imaging (MRI) and positron emission tomography-computed tomography (PET-CT) on Oct 8, 2015 for the 5-year-old girl with multiple posterior fossa lesions. A Axial gadolinium-enhanced MRI displays a lesion $(2.0 \mathrm{~cm} \times 2.6 \mathrm{~cm}$; white arrow) in the cerebello-pontine angle (CPA). No relatively clear margin was observed between the lesion and the pons. B Sagittal gadolinium-enhanced MRI shows two lesions. The upper left pontine lesion $(0.5 \mathrm{~cm} \times 0.6 \mathrm{~cm})$ was significantly gadolinium-enhanced with relatively clear margin (red arrow). From the sagittal view, the CPA lesion (white arrow) seemingly demonstrates clear boundary to the pons. C Magnetic resonance spectroscopy (MRS) of the lesion shows a decrease in choline (Cho) and N-acetyl aspartate (NAA). D PET-CT image demonstrates a hypometabolic lesion (white arrow) in the left side of the CPA 
The patient showed weak expression of serum antibodies for tubercle bacillus (TB). The results of sputum smear examination and blood interferon-gamma release assay for the detection of TB infection were negative. Serum levels of tumor markers [including alpha-fetoprotein, carcinoembryonic antigen, carbohydrate antigen (CA)-724, CA-125, CA-199, and squamous cell carcinoma antigen] were within normal ranges, except for a slight increase in the level of neuron-specific enolase. The cerebrospinal fluid was extracted via lumbar puncture and tested for TB-DNA and TB-RNA, yielding negative results. After consultation with a tuberculosis specialist, the current evidence for intracranial tuberculoma was deemed insufficient. We proposed a biopsy of the left CPA lesion, but the patient's parents refused and demanded discharge.

Two months later, the patient underwent another MRI. No involvement of additional nerves was observed, except for the cranial VI palsy. However, progression of the disease was demonstrated: (1) the left CPA lesion was enlarged and extended along the cistern of CPA (Fig. 2A, $\mathrm{B}$, white arrow); (2) the upper left pontine lesion was enlarged (Fig. 2B, red arrow); and most importantly, (3) a new lesion had emerged in the upper right side of the pons (Fig. 2A, yellow arrow). In addition, MRS revealed a radical decrease of NAA and a drastic increase of Cho with an obvious increase in the Cho:NAA ratio (Cho:NAA $=1.56)$ and a decrease in the Cho: $\mathrm{Cr}$ ratio (Cho:Cr $=11.87$ ) (Fig. $2 \mathrm{C}$ ). A peak at $1.3 \mathrm{ppm}$ was displayed. We strongly suggested surgery for pathologic diagnosis and follow-up treatment as soon as possible, but the parents refused again.

One month later, the patient was admitted again because of malnutrition and weakness with frequent vomiting. Re-examination with MRI demonstrated progression of all three lesions, especially the CPA lesion, which progressed and expanded to the anterior CPA cistern (Fig. 2D, E). MRS data showed the same pattern as the previous examination, with a significantly increased
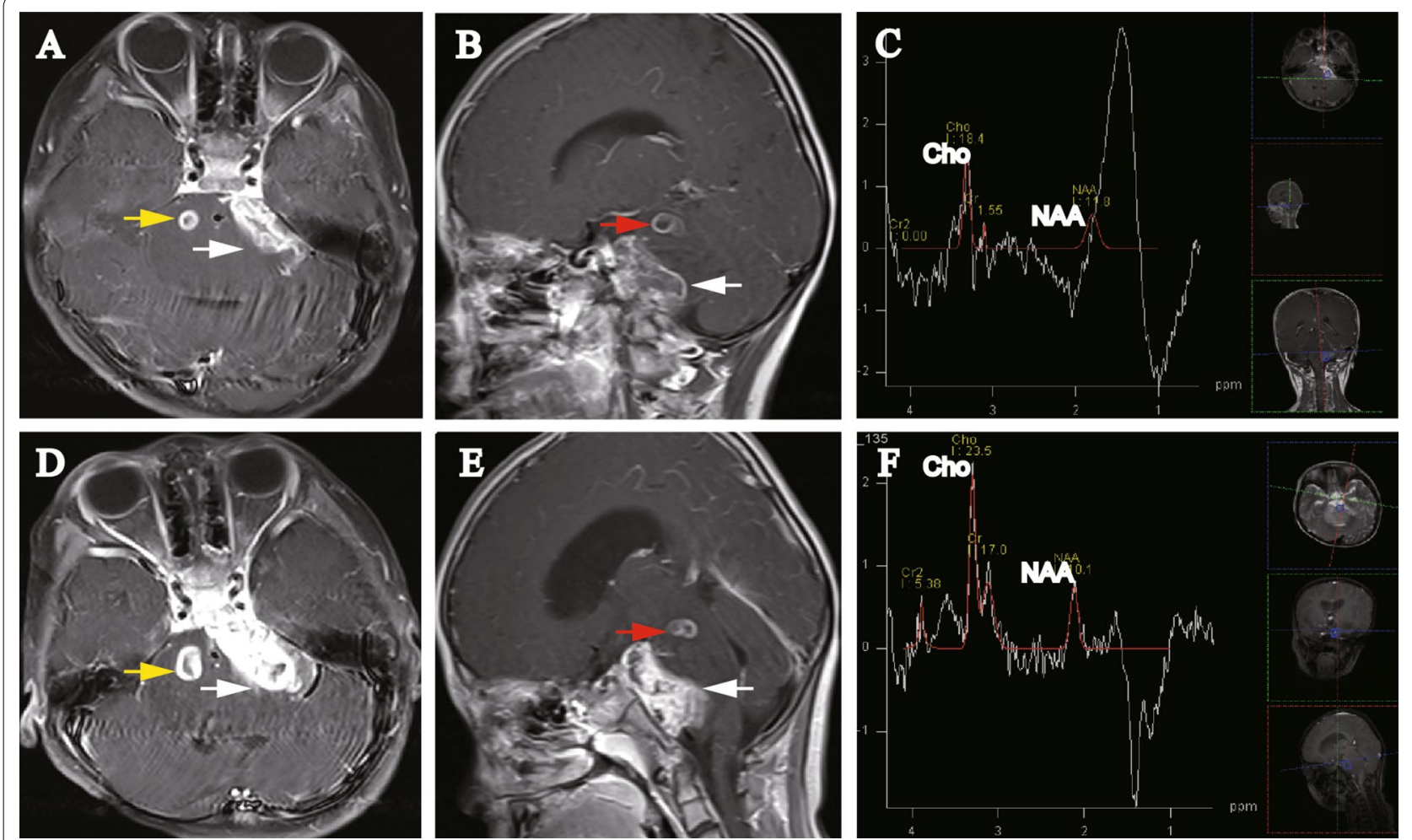

Fig. 2 Images of repeated MRI on Dec 11, 2015 and Jan 12, 2016 for the 5-year-old girl with multiple posterior fossa lesions. A Axial gadoliniumenhanced MRI (Dec 11, 2015) displays the occurrence of a new lesion $(0.8 \mathrm{~cm} \times 0.9 \mathrm{~cm}$, yellow arrow). The left CPA lesion is enlarged and extended along the cistern of the CPA $(3.1 \mathrm{~cm} \times 1.2 \mathrm{~cm}$, white arrow). B Sagittal gadolinium-enhanced MRI (Dec 11, 2015) shows that the CPA lesion was enlarged and extended along the cistern of CPA (white arrow) and the upper left pontine lesion was enlarged (red arrow). C MRS (Dec 11, 2015) shows increased Cho and decreased NAA expression. The Cho:NAA ratio was also significantly increased. D Axial gadolinium-enhanced MRI (Jan 12, 2016) displays that both the CPA lesion (white arrow) and the new lesion in the right side of the pons are enlarged (1.1 cm $\times 1.3 \mathrm{~cm}$, yellow arrow). The CPA lesion is extended along the cistern of the CPA. E Sagittal gadolinium-enhanced MRI (Jan 12, 2016) shows that both the CPA lesion (white arrow) and upper left pontine lesion (red arrow) were enlarged. F MRS (Jan 12, 2016) shows further increased Cho and decreased NAA expression. The Cho:NAA ratio was 2.33 
Cho:NAA ratio (Cho:NAA $=2.33$ ) and dramatically decreased Cho: $\mathrm{Cr}$ ratio (Cho: $\mathrm{Cr}=1.38$ ) (Fig. $2 \mathrm{~F}$ ). A reverse peak at $1.3 \mathrm{ppm}$ was shown. The parents finally agreed to the suggested surgery.

\section{Surgery and pathology}

Histopathologic diagnosis and safe resection of the lesion were the goals of the suggested surgery. Under these circumstances, the CPA lesion was the target of the operation, whereas the other two lesions were left untouched. The surgery was conducted using a suboccipital retrosigmoid approach. After occipital craniotomy and dura opening, the cerebellum was cautiously separated from the CPA, and the cerebrospinal fluid was slowly released and suctioned. The arachnoid of the CPA was opened, and a yellowish nodule-like mass was encountered with a clear boundary to the cerebellum (Fig. 3a). The vessels distributed on the surface of the lesion were carefully coagulated, and the lesion was moderately rubbery. The tissue was formalin-fixed and paraffin-embedded for a pathologic examination with hematoxylin-eosin staining, which indicated moderate mitoses, necrosis, and vascularization, suggesting low-grade glioma (Fig. 3b, c). Resection of the lesion combined with ventricular peritoneal drainage was performed. The internal portion of the lesion, with a relatively higher blood supply, was softer than the outer layer (Fig. 3d). No clear boundary was found between the lesion and the pons or the root entry zone of the trigeminal nerve. Histopathologic examination revealed a highly malignant tumor with marked necrosis and vascularization (Fig. 3e, f). No typical pseudopalisading signs or glomerular structures were observed in this case. The internal portion of the CPA lesion was removed within the arachnoid boundary until neurophysiological monitoring of the caudal cranial nerves displayed a slightly decreasing reaction. Postoperative MRI confirmed the subtotal resection of the CPA lesion. No further surgery-related neurological deficits occurred.

The final pathologic diagnosis was glioblastoma with diffuse and strong expression of glial fibrillary acid protein (GFAP, Fig. 4a) and S-100 (Fig. 4b). The positive rates were $80 \%-90 \%$ for both P53 (Fig. 4c) and Ki-67 (Fig. 4d) and $1 \%$ for methylated O6-methylguanine-DNA methyltransferase (Fig. 4e). The expression of isocitrate dehydrogenase 1 (IDH1) was weak (Fig. 4f).

The patient received no further chemotherapy or radiotherapy and died 2 months later.

\section{Discussion}

The preoperative diagnosis of this case was difficult. Brain metastases, central nervous system (CNS) lymphomas, and inflammatory disease were considered in the differential diagnosis. It is notable that the patient
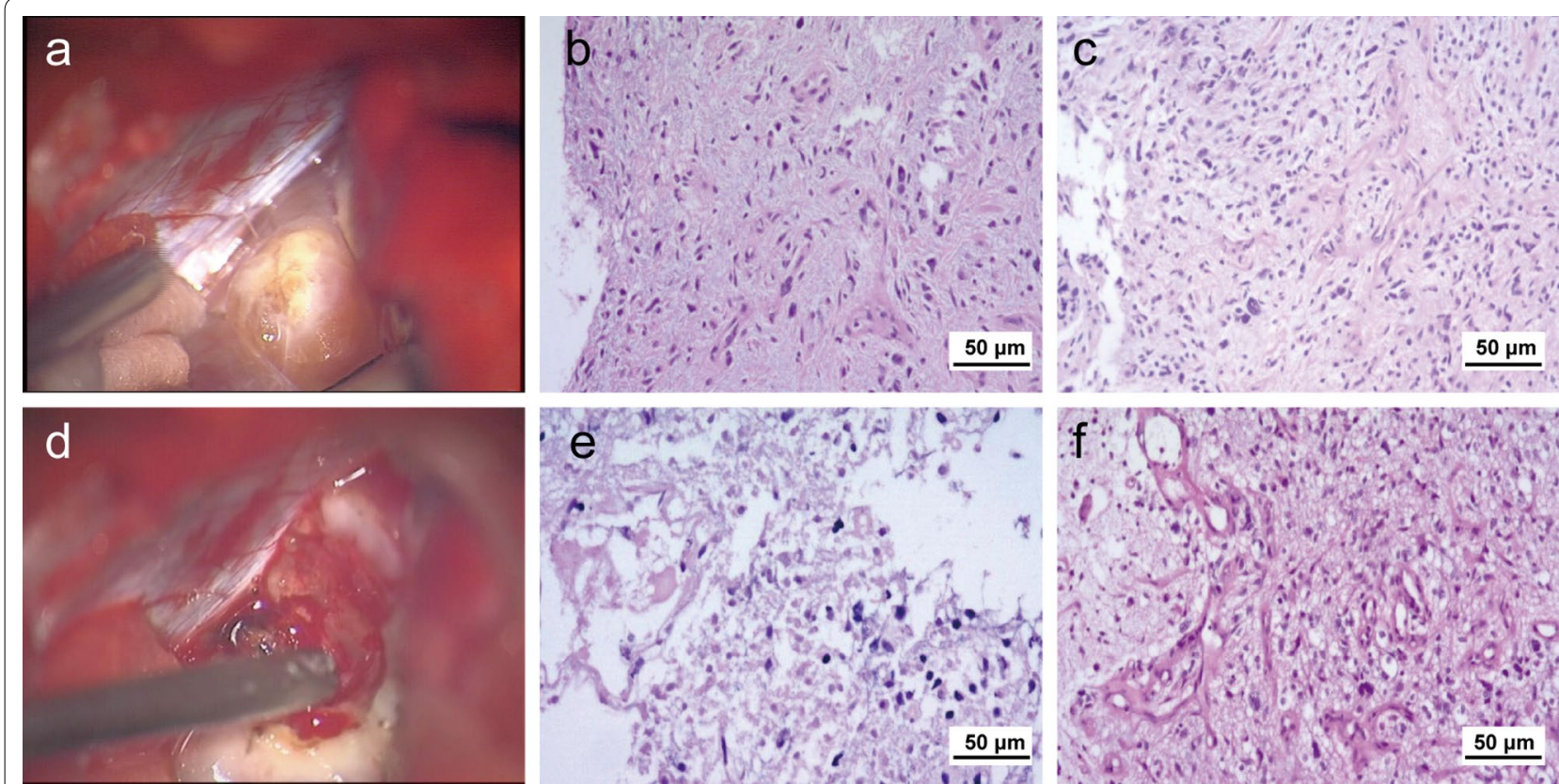

Fig. 3 Histopathologic examination of the CPA lesion with hematoxylin-eosin staining. a Macroscopically, the outer layer of CPA lesion is rubbery and nodule-like. $\mathbf{b}$ Under a microscope, the outer layer of the CPA lesion displays moderate mitoses and necrosis. $\mathbf{c}$ Under a microscope, the outer layer of the CPA lesion displays moderate vascularization. d Macroscopically, the internal portion of the lesion is softer and has a higher blood supply than the outer layer. e Under a microscope, the internal portion of the CPA lesion displays obvious mitoses and necrosis. $\mathbf{f}$ Under a microscope, the internal portion of the CPA lesion displays hyper-vascularization 

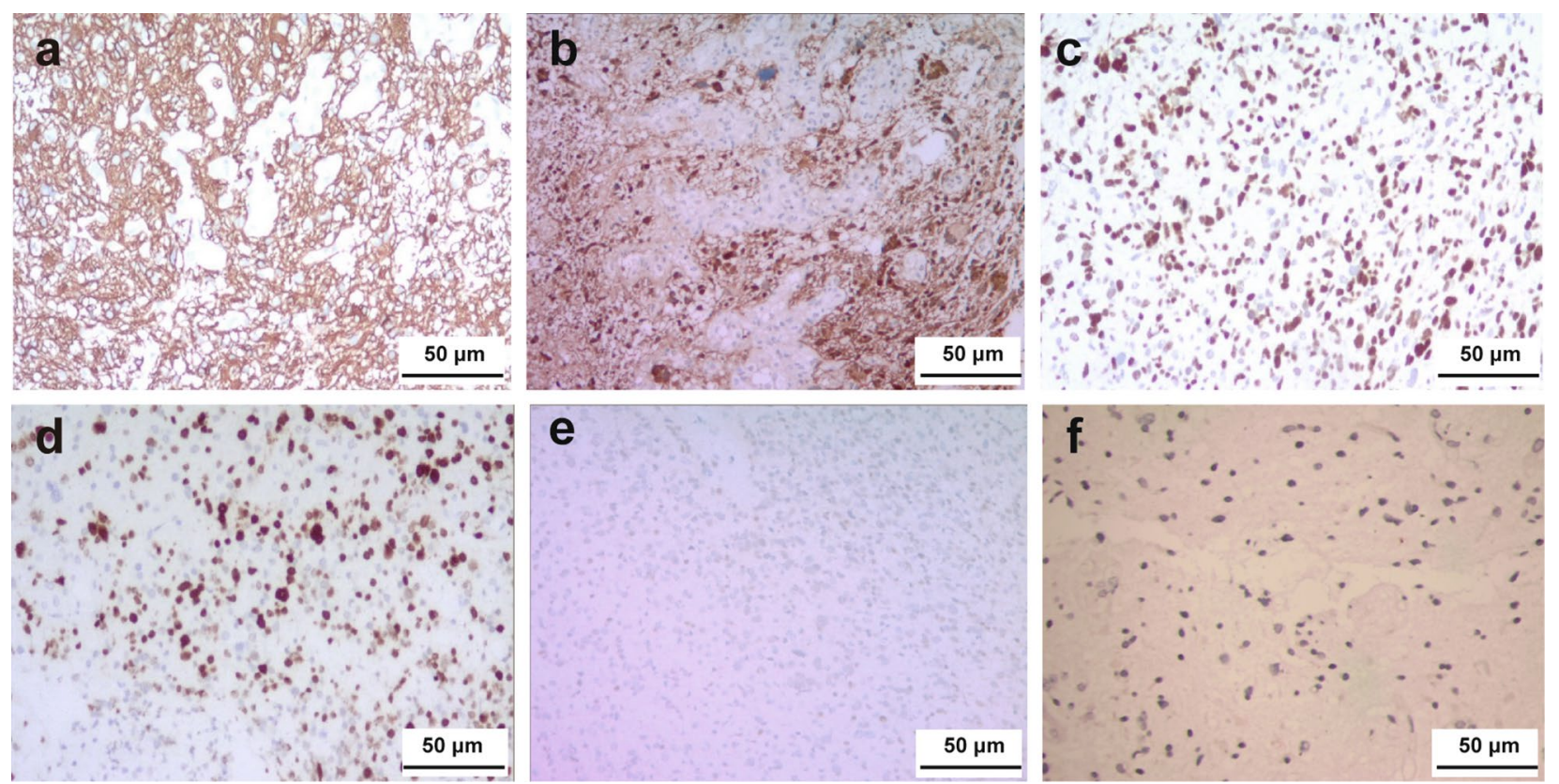

Fig. 4 Immunohistochemical examination of the tumor with diaminobenzidine staining. Glial fibrillary acidic protein (a), S-100 (b), P53 (c), and Ki-67 (d) are diffusely expressed. The expression of methylated O6-methylguanine-DNA methyltransferase (e) and isocitrate dehydrogenase $1(\mathbf{f})$ is weak

presented with frequent fever and cough and had weak positivity for blood TB antibodies. Infectious diseases, such as tuberculosis, were considered. Although previous articles mentioned that ring-like enhancement and multiple lesions are indications of posterior fossa glioblastoma [7], these neurological imaging features are frequently observed in tuberculoma and tuberculous lesions that are occasionally secondary to tuberculous arachnoiditis [8]. Surgical resection or biopsy is necessary for a definitive diagnosis. The histological grading is based on the degree of anaplasia and dedifferentiation, number of mitoses, capillary endothelial proliferation, and presence of necrosis [9-11].

Immunohistochemical examination showed diffuse expression of GFAP, P53, and S-100 in our case. The expression of S-100 is not stable in glioma, whereas GFAP is a qualitative diagnostic marker of glioma [12]. The combination of all histopathologic evidence supported the diagnosis of glioblastoma in our case.

The progressive growth pattern of the CPA lesion is a confusing characteristic of this case, especially given the coexistence of multiple lesions. We searched PubMed and Google Scholar and found only two English-language articles reporting the coexistence of multiple lesions and a CPA lesion [13, 14]. According to the literature, CPA glioblastomas may have different origins [15]. Wu et al. [16] reported a CPA glioblastoma that originated from the cranial VIII nerve and reviewed seven other similar cases. Cranial nerve glioblastomas are presumably originated from CNS tissue locating at the root entry zone of the cranial nerve or from heterotopic neuroglia cells in the leptomeninges. Similarly, Mabray et al. [17] recently reported eight cases of glioblastoma originated from the cranial nerve. These glioblastomas were located at the CPA and involved the unilateral brainstem. The cerebellum is another unusual place for glioblastoma development. Matsuda et al. [18] reported a case of glioblastoma originated from the cerebellum and reviewed another three similar cases. In the reports by both Mabray et al. [17] and Matsuda et al. [18], the relatively clear boundary between the brainstem and tumor were demonstrated during the operation. In our case, neither preoperative MRI nor surgery alone supported an origin at the cranial nerve cerebellum. However, no clear boundary between the CPA lesion and the pons was discovered during our surgical procedure. This evidence, along with MRI findings on the CPA lesions and two pontine lesions, confirmed the diagnosis of primary exophytic pontine glioblastoma. The T2-weighted image showed that the CPA lesion was confined to the pons, with an outer layer that consisted of edematous tumor tissue infiltrating the pons. This finding may explain that the pathologic presentation of the outer layer of the tumor was not as typical for glioblastoma as that of the inner part of the tumor, emphasizing the diverse pathologic features of different parts of the tumor and the need for accurate and 
comprehensive specimen collection in such a case. As reported earlier, the biopsy samples should be collected from solid, non-necrotic, and gadolinium-enhanced areas, if possible [19].

According to the imaging and histopathologic evidence, this case of pontine glioblastoma can be categorized as a brainstem glioma. However, the classification of brainstem gliomas is not uniform. Choux et al. [20] classified brainstem tumors as diffuse glioma, focal intrinsic glioma (solid or cystic), exophytic glioma, and cervicomedullary glioma. Fisher et al. [21] anatomically classified brainstem glioma as diffuse intrinsic pontine glioma (DIPG), exophytic medullary glioma, and midbrain or tectal glioma. However, they also considered it was reasonable to categorize brainstem tumors simply as diffuse or focal glioma. Our case possessed some typical features of DIPG, such as an infiltrative lesion occupying $50 \%$ of the pons, obvious expansion of the pons, and engulfment of the basilar artery as reported in the literature $[22,23]$. DIPG may extend laterally into the cerebellar peduncles, cerebellar hemispheres, midbrain, and medulla [24], whereas the exophytic growth to the CPA is not a common feature of typical DIPG. This situation may explain why some researchers exclude exophytic pontine glioma from the diagnostic criteria of DIPG [22, 25]. Given that various manifestations of DIPG are often observed, the term "atypical DIPG" was adopted by some researchers [26]. It seems that our case may be classified as diffuse brainstem (pontine) glioma or an atypical subtype of DIPG (Table 1).

The changes in the MRS results of our case merit attention. It was reported that the Cho:NAA ratio calculated according to MRS data can be used to monitor metabolism and glioma behavior [22]. In pediatric CNS tumors, changes in levels of metabolic markers as determined with MRS are associated with tumor grade [27]. Furthermore, an increasing Cho:NAA ratio predicts a short overall survival $[22,28]$. PET measures the regional cerebral glucose metabolism, offering valuable information that can be used to differentiate between low-grade and high-grade gliomas [29-31]. For our case, the initial MRS results (obtained on October 8, 2015) showed a simultaneous decrease in NAA and Cho and an increased Cho:NAA ratio, leading to the diagnosis of low-grade glioma. The decrease in NAA, Cho, and Cho:NAA ratio of the first MRS may be technique variations such as the selection bias of regions of interests. Taking both MRS and PET results into consideration, a diagnosis of low-grade glioma seems reasonable. Earlier PET findings from another medical center were consistent with our initial MRS results. Repeated MRS performed 2 and 3 months later demonstrated an obvious decrease in NAA and an increase in Cho, indicating a high-grade glioma. In addition, we noticed peaks at approximately 1.3 ppm (Figs. 1c, 2c, f). Two situations were considered: (1) technical problems such as unstable baseline or lipid effection and (2) a lac-lip peak demonstrating necrosis or hypoxia. Although necrosis is usually found in high-grade glioma, PET-CT results and the increasing tendency of the Cho:NAA ratio both support the initial diagnosis of low-grade glioma. The MRS characteristics of this tumor seemed to be significantly altered within merely 2 months according to consecutive neurological imaging.

Glioblastomas are divided into two types of genesis patterns: primary glioblastoma, also termed de novo glioblastoma, which is defined as a fully developed tumor with no clinical, radiological, or histopathologic evidence of relative benign precursor lesion [32-34], and secondary glioblastoma progressing from low-grade astrocytoma, as supported by imaging or histological evidence $[32,33,35]$. According to the literature, the proportions of primary and secondary glioblastomas are approximately $90 \%$ and $10 \%$, respectively [36]; the disease course of primary glioblastomas was less than 3 months, whereas the progression from relative low-grade astrocytoma to glioblastoma took approximately 2-5 years [37]. Nevertheless, rapidly progressive cases of secondary glioblastoma have been reported [34]. Furthermore, as for brainstem gliomas, it has occasionally been reported that low-grade gliomas behaved as aggressively as highgrade gliomas [38, 39]. Recent studies have revealed the histological heterogeneity of DIPG. Based on the autopsy results of the patients diagnosed with DIPG, It was reported that the primary and metastatic lesions displayed high and low histological grades, respectively [40, 41]. Accordingly, DIPG was classified as primary lesions, contiguous lesions, and metastatic lesions according to the locations [41]. The different pathologic manifestations of the two samples from our case may be related to the differences of "central" and "marginal" areas of the lesion. This may again emphasize the importance of accurate sampling.

Immunohistochemical examination of our case demonstrated a high positive rate of P53 (80\%-90\%), which is a frequently expressed marker of DIPG [42]. It was also reported that P53 mutation was even more frequently and increasingly expressed in secondary glioblastomas, which was in contrast with its rare expression in primary glioblastoma [34]. However, IDH1, a biomarker of secondary glioblastoma [43], showed low expression in our case. This may highlight the molecular differences between pediatric and adult secondary glioblastomas [44-46]. In children, low expression of IDH1 may be present in secondary glioblastomas. According to the literature, histological or imaging evidence of a progression from a less malignant astrocytoma can be used as 


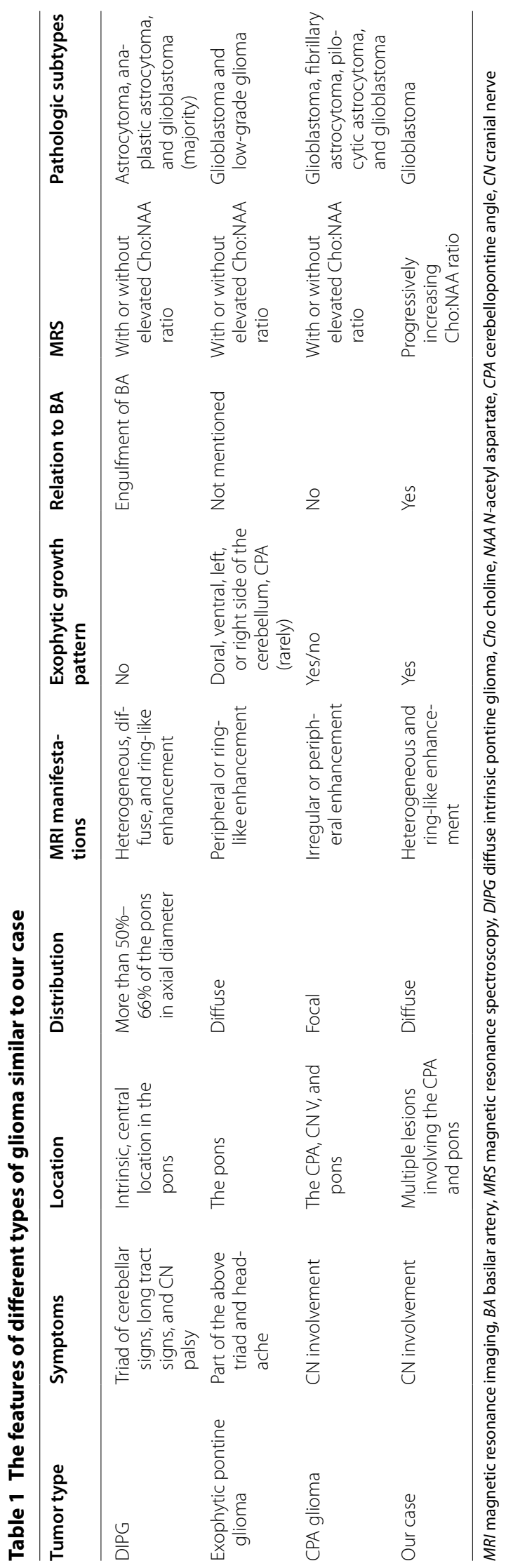


a diagnostic criterion of secondary glioblastomas [34]. Based on this knowledge and results of repeated MRI (MRS), in combination with initial PET-CT findings, we were inclined to diagnose our case as a rapidly progressive secondary glioblastoma.

Multiple gadolinium-enhanced lesions are rarely seen in brainstem gliomas [47-49]. Multiple-lesion gliomas involve at least two enhanced lesions and account for $2 \%-9 \%$ of all gliomas [50-52]. This rare entity is classified into two distinct types, multifocal and multicentric gliomas. Multifocal gliomas possess homology or dissemination of definite pathways. Multicentric gliomas are defined as anatomically isolated lesions and are usually distributed at different lobes or hemispheres of the brain [51]. Multiple lesions can be present at the diagnosis or at a progressive stage for both multicentric and multifocal gliomas [53]. In our case, the right pontine lesion was developed at a later stage as compared with the other two lesions, and all lesions were located at the diffusely swelling pons, indicating a diagnosis of multifocal glioblastoma rather than multicentric glioblastoma.

Diffuse pontine glioma is the most common type of brainstem tumor and carries the poorest prognosis in children [23]. Early surgical intervention to determine the diagnosis is recommended [54-56]. The standard treatment of DIPG is external beam radiation therapy administered in fractions over approximately 6 weeks at a total dose of 60 Gy [54]. Diffuse intrinsic pontine glioma is almost invariably fatal with a mean patient overall survival of 9-12 months from the time of diagnosis [57]. Radiation therapy is the major treatment [58]. Adjuvant therapies, such as chemotherapy, targeted therapy, differentiation agents, and radiation sensitizers, have been studied. Although some of these therapies showed a beneficial impact on patient outcomes $[25,58]$, further research is required.

\section{Conclusion}

In summary, we presented a rare case of progressive, exophytic, multifocal pontine glioblastoma in a 5-year-old girl. A preoperative differential diagnosis was difficult because of the atypical neurological imaging features and frequent fever and cough symptoms. The clinical evidence supported a secondary glioblastoma. Regretfully, an early histopathologic diagnosis was not achieved.

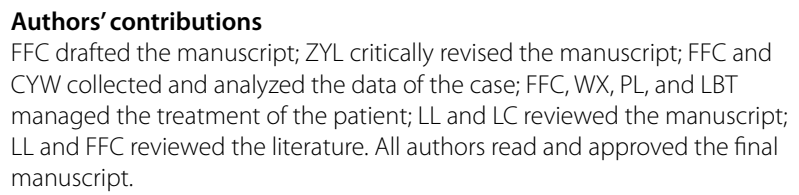

FFC drafted the manuscript; ZYL critically revised the manuscript; FFC and CYW collected and analyzed the data of the case; FFC, WX, PL, and LBT managed the treatment of the patient; $L L$ and $L C$ reviewed the manuscript; $\mathrm{LL}$ and FFC reviewed the literature. All authors read and approved the final manuscript.

\section{Author details}

${ }^{1}$ Neurosurgery Department, Guangzhou First People's Hospital, Guangzhou Medical University, Guangzhou 510180, Guangdong, P. R. China.
${ }^{2}$ Neurosurgery Department, Shenzhen Second People's Hospital, Shenzhen University, Shenzhen 518000, Guangdong, P. R. China. ${ }^{3}$ Oncology Department, Guangzhou First People's Hospital, Guangzhou Medical University, Guangzhou 510180, Guangdong, P. R. China. ${ }^{4}$ Record Department, Guangzhou First People's Hospital, Guangzhou Medical University, Guangzhou 510180, Guangdong, P. R. China.

\section{Competing interests}

The authors declare that they have no competing interests.

Received: 13 May 2016 Accepted: 3 January 2017

Published online: 27 March 2017

References

1. Rubin G, Michowitz S, Horev G, Herscovici Z, Cohen IJ, Shuper A et al. Pediatric brain stem gliomas: an update. Childs Nerv Syst. 1998;14(4-5):167-73.

2. Freeman CR, Farmer JP. Pediatric brain stem gliomas: a review. Int J Radiat Oncol Biol Phys. 1998;40(2):265-71.

3. Tomita T, Grahovac G. Cerebellopontine angle tumors in infants and children. Childs Nerv Syst. 2015;31(10):1739-50.

4. Holman MA, Schmitt WR, Carlson ML, Driscoll CL, Beatty CW, Link MJ. Pediatric cerebellopontine angle and internal auditory canal tumors: clinical article. J Neurosurg Pediatr. 2013;12(4):317-24.

5. Ragab A, Emara A, Shouker M, Ebied O. Prospective evaluation of the clinical profile and referral pattern differences of vestibular schwannomas and other cerebellopontine angle tumors. Otol Neurotol. 2012;33(5):863-70.

6. Phi JH, Wang KC, Kim IO, Cheon JE, Choi JW, Cho BK, et al. Tumors in the cerebellopontine angle in children: warning of a high probability of malignancy. J Neurooncol. 2013;112(3):383-91.

7. Kuroiwa T, Numaguchi Y, Rothman Ml, Zoarski GH, Morikawa M, Zagardo MT, et al. Posterior fossa glioblastoma multiforme: MR findings. Am J Neuroradiol. 1995;16(3):583-9.

8. Shingade RG, Prakashchandra SP. Role of advanced diagnostic imaging in intracranial tuberculoma: MR spectroscopy. J Clin Diagn Res. 2015:9(8):TJ03-4.

9. Kane AG, Robles HA, Smirniotopoulos JG, Heironimus JD, Fish MH. Radiologic-pathologic correlation. Diffuse pontine astrocytoma. Am J Neuroradiol. 1993;14(4):941-5

10. Stroink AR, Hoffman HJ, Hendrick EB, Humphreys RP, Davidson G. Transependymal benign dorsally exophytic brain stem gliomas in childhood: diagnosis and treatment recommendations. Neurosurgery. 1987;20(3):439-44.

11. Stroink AR, Hoffman HJ, Hendrick EB, Humphreys RP. Diagnosis and management of pediatric brain-stem gliomas. J Neurosurg. 1986;65(6):745-50.

12. Suzuki H, Uenohara H, Utsunomiya A, Kurihara N, Suzuki S, Tadokoro M, et al. A case of angioglioma composed of astrocytoma with a papillary growth pattern: immunohistochemical and ultrastructural studies. Brain Tumor Pathol. 2002;19(2):111-6.

13. Bussone G, Sinatra MG, Boiardi A, Lazzaroni M, Mariani C, Allegranza A. A case of glioblastoma with multiple centers above and below the tentorium. J Neurooncol. 1979;221(3):187-92.

14. Kasliwal MK, Gupta DK, Mahapatra AK, Sharma MC. Multicentric cerebellopontine angle glioblastoma multiforme. Pediatr Neurosurg. 2008;44(3):224-8

15. Breshears JD, Ivan ME, Cotter JA, Bollen AW, Theodosopoulos PV, Berger MS. Primary glioblastoma of the trigeminal nerve root entry zone: case report. J Neurosurg. 2015;122(1):78-81.

16. Wu B, Liu W, Zhu H, Feng H, Liu J. Primary glioblastoma of the cerebellopontine angle in adults. J Neurosurg. 2011;114(5):1288-93.

17. Mabray MC, Glastonbury CM, Mamlouk MD, Punch GE, Solomon DA, Cha S. Direct cranial nerve involvement by gliomas: case series and review of the literature. Am J Neuroradiol. 2015;36(7):1349-54.

18. Matsuda M, Onuma K, Satomi K, Nakai K, Yamamoto T, Matsumura A. Exophytic cerebellar glioblastoma in the cerebellopontine angle: case report and review of the literature. J Neurol Surg Rep. 2014;75(1):e67-72. 
19. Kwon JW, Kim IO, Cheon JE, Kim WS, Moon SG, Kim TJ, et al. Paediatric brain-stem gliomas: MRI, FDG-PET and histological grading correlation. Pediatr Radiol. 2006;36(9):959-64.

20. Choux M, Lena G, Do L. Brainstem tumors. In: Choux M, Di Rocco C, Hockley A, et al., editors. Pediatric neurosurgery. New York: Churchill Livingstone; 2000. p. 471-91.

21. Fisher PG, Breiter SN, Carson BS, Wharam MD, Williams JA, Weingart JD, et al. A clinicopathologic reappraisal of brain stem tumor classification. Identification of pilocystic astrocytoma and fibrillary astrocytoma as distinct entities. Cancer. 2000;89(7):1569-76.

22. Steffen-Smith EA, Shih JH, Hipp SJ, Bent R, Warren KE. Proton magnetic resonance spectroscopy predicts survival in children with diffuse intrinsic pontine glioma. J Neurol. 2011;105(2):365-73.

23. Fischbein NJ, Prados MD, Wara W, Russo C, Edwards MS, Barkovich AJ. Radiologic classification of brain stem tumors: correlation of magnetic resonance imaging appearance with clinical outcome. Pediatr Neurosurg. 1996;24(1):9-23.

24. Bartels U, Hawkins C, Vezina G, Kun L, Souweidane M, Bouffet E. Proceedings of the diffuse intrinsic pontine glioma (dipg) toronto think tank: advancing basic and translational research and cooperation in dipg. $J$ Neurol. 2011;105(1):119-25.

25. Grimm SA, Chamberlain MC. Brainstem glioma: a review. Curr Neurol Neurosci Rep. 2013;13(5):346.

26. Hankinson TC, Campagna EJ, Foreman NK, Handler MH. Interpretation of magnetic resonance images in diffuse intrinsic pontine glioma: a survey of pediatric neurosurgeons. J Neurosurg Pediatr. 2011;8(1):97-102.

27. Peet AC, Lateef S, MacPherson L, Natarajan K, Sgouros S, Grundy RG. Short echo time $1 \mathrm{~h}$ magnetic resonance spectroscopy of childhood brain tumours. Childs Nerv Syst. 2007;23(2):163-9.

28. Hipp SJ, Steffen-Smith E, Hammoud D, Shih JH, Bent R, Warren KE. Predicting outcome of children with diffuse intrinsic pontine gliomas using multiparametric imaging. Neuro-oncology. 2011;13(8):904-9.

29. Delbeke D, Meyerowitz C, Lapidus RL, Maciunas RJ, Jennings MT, Moots $\mathrm{PL}$, et al. Optimal cutoff levels of $\mathrm{f}$-18 fluorodeoxyglucose uptake in the differentiation of low-grade from high-grade brain tumors with pet. Radiology. 1995;195(1):47-52.

30. Padma MV, Said S, Jacobs M, et al. Prediction of pathology and survival by FDG-PET in gliomas. J Neurol. 2003;64(3):227-37.

31. De Witte O, Lefranc F, Levivier M, Salmon I, Brotchi J, Goldman S. FDG-PET as a prognostic factor in high-grade astrocytoma. J Neurol. 2000;49(2):157-63.

32. Kleihues P, Ohgaki H. Phenotype vs genotype in the evolution of astrocytic brain tumors. Toxicol Pathol. 2000;28(1):164-70.

33. Ohgaki $\mathrm{H}$, Kleihues P. Genetic alterations and signaling pathways in the evolution of gliomas. Cancer Sci. 2009;100(12):2235-41.

34. Ohgaki H, Kleihues P. Genetic pathways to primary and secondary glioblastoma. Am J Pathol. 2007;170(5):1445-53.

35. Ohgaki H, Dessen P, Jourde B, Horstmann S, Nishikawa T, Di Patre PL, et al. Genetic pathways to glioblastoma: a population-based study. Cancer Res. 2004;64(19):6892-9.

36. Tso CL, Freije WA, Day A, Chen Z, Merriman B, Perlina A, et al. Distinct transcription profiles of primary and secondary glioblastoma subgroups. Cancer Res. 2006;66(1):159-67.

37. Ohgaki H, Kleihues P. Population-based studies on incidence, survival rates, and genetic alterations in astrocytic and oligodendroglial gliomas. J Neuropathol Exp Neurol. 2005;64(6):479-89.

38. Cage TA, Samagh SP, Mueller S, Nicolaides T, Haas-Kogan D, Prados M, et al. Feasibility, safety, and indications for surgical biopsy of intrinsic brainstem tumors in children. Childs Nerv Syst. 2013;29(8):1313-9.
39. Yoshimura J, Onda K, Tanaka R, Takahashi H. Clinicopathological study of diffuse type brainstem gliomas: analysis of 40 autopsy cases. Neurol Med Chir (Tokyo). 2003;43(8):375-82.

40. Buczkowicz P, Bartels U, Bouffet E, Becher O, Hawkins C. Histopathological spectrum of paediatric diffuse intrinsic pontine glioma: diagnostic and therapeutic implications. Acta Neuropathol. 2014;128:573-81.

41. Hoffman LM, DeWire M, Ryall S, Buczkowicz P, Leach J, Miles L, et al. Spatial genomic heterogeneity in diffuse intrinsic pontine and midline high-grade glioma: implications for diagnostic biopsy and targeted therapeutics. Acta Neuropathol Commun. 2016;4(4):1.

42. Ballester LY, Wang Z, Shandilya S, Miettinen M, Burger PC, Eberhart CG, et al. Morphologic characteristics and immunohistochemical profile of diffuse intrinsic pontine gliomas. Am J Surg Pathol. 2013;37(9):1357-64.

43. Hofer S, Rushing E, Preusser M, Marosi C. Molecular biology of high-grade gliomas: what should the clinician know? Chin J Cancer. 2014;33(1):4-7.

44. Paugh BS, Qu C, Jones C, Liu Z, Adamowicz-Brice M, Zhang J, et al. Integrated molecular genetic profiling of pediatric high-grade gliomas reveals key differences with the adult disease. J Clin Oncol. 2010;28(18):3061-8.

45. Oka H, Utsuki S, Tanizaki Y, Hagiwara H, Miyajima Y, Sato K, et al. Clinicopathological features of human brainstem gliomas. Brain Tumor Pathol. 2013;30(1):1-7.

46. Grill J, Puget S, Andreiuolo F, Philippe C, MacConaill L, Kieran MW. Critical oncogenic mutations in newly diagnosed pediatric diffuse intrinsic pontine glioma. Pediatr Blood Cancer. 2012;58(4):489-91.

47. Salunke P, Sura S, Tewari MK, Gupta K, Khandelwal NK. An exophytic brain stem glioblastoma in an elderly presenting as a cerebellopontine angle syndrome. Br J Neurosurg. 2012;26(1):96-8.

48. Arnautovic Kl, Husain MM, Linskey ME. Cranial nerve root entry zone primary cerebellopontine angle gliomas: a rare and poorly recognized subset of extraparenchymal tumors. J Neurol. 2000;49(3):205-12.

49. Luetjens G, Mirzayan MJ, Brandis A, Krauss JK. Exophytic giant cell glioblastoma of the medulla oblongata. J Neurosurg. 2009;110(3):589-93.

50. Barnard RO, Geddes JF. The incidence of multifocal cerebral gliomas. A histologic study of large hemisphere sections. Cancer. 1987;60(7):1519-31.

51. Batzdorf U, Malamud N. The problem of multicentric gliomas. J Neurosurg. 1963;20:122-36.

52. Kyritsis AP, Yung WK, Leeds NE, Bruner J, Gleason MJ, Levin VA. Multifocal cerebral gliomas associated with secondary malignancies. Lancet. 1992;339(8803):1229-30.

53. Kyritsis AP, Levin VA, Yung WK, Leeds NE. Imaging patterns of multifocal gliomas. Eur J Radiol. 1993;16(3):163-70.

54. Vanan MI, Eisenstat DD. DIPG in children-what can we learn from the past? Front Oncol. 2015;5:237.

55. Villa S, Balana C, Comas S. Radiation and concomitant chemotherapy for patients with glioblastoma multiforme. Chin J Cancer. 2014;33(1):25-31.

56. Puget S, Beccaria K, Blauwblomme T, Roujeau T, James S, Grill J, et al. Biopsy in a series of 130 pediatric diffuse intrinsic Pontine gliomas. Childs Nerv Syst. 2015;31(10):1773-80.

57. Laigle-Donadey F, Doz F, Delattre J-Y. Brainstem gliomas in children and adults. Curr Opin Oncol. 2008;20(6):662-7.

58. Warren KE. Diffuse intrinsic pontine glioma: poised for progress. Front Oncol. 2012;2:205. 